\title{
SHAKING TABLE TESTS ON UNREINFORCED LOAD-BEARING MASONRY WALLS - COMPARISON WITH SIMPLE ROCKING MODELS
}

\author{
Christophe Mordant ${ }^{1}$, Matt Dietz ${ }^{2}$, Hervé Degée ${ }^{1}$ \\ ${ }^{1}$ University of Liège \\ Chemin des Chevreuils, 1 - 4000 Liège (Belgium) \\ cmordant@ulg.ac.be / h.degee@ulg.ac.be \\ ${ }^{2}$ University of Bristol \\ University Walk, Bristol BS8 1TR (UK) \\ m.dietz@bristol.ac.uk
}

Keywords: Unreinforced masonry walls, Rocking, Coefficient of restitution

\begin{abstract}
Assessment of the overall seismic performances of multi-storey unreinforced masonry structures requires an appropriate characterization of the behaviour of their structural components, in particular when these are subjected to a dynamic ground motion input. In order to develop a better understanding on this issue and in a further perspective of investigating the consequences of the presence of $1 \mathrm{~cm}$ thick rubber elements used for improving the sound-proofing performances of the building, shaking table tests have been carried out in the framework of the European project SERIES. Four single walls were tested. These were built with high resistance thin-bed layered clay masonry with empty vertical joints. Two of them had an aspect ratio close to 1, while the other two were close to 0.4. One wall of each aspect ratio included rubber devices at its bottom and top to enable comparisons and conclusions about the influence of rubber on the wall behaviour. The test results were then partially compared to results obtained with a theoretical rocking model considering the wall as a rigid body. The results summarized in the present contribution evidence a significant rocking behaviour for the highest input acceleration levels. Characterization of this behaviour is however strongly dependent on the aspect ratio of the wall and on the presence or not of rubber devices in terms of natural frequencies, damping, dynamic amplifications and progressive damage with increasing acceleration levels. It is also showed that the theoretical rocking predictions are in good agreement with the experimental results for high acceleration levels, while the behaviour is closer to the one of a cantilever for the lower levels. It is finally evidenced that, in presence of acoustic rubber devices, amplitudes of the rocking motion are increased but with a more limited damaging of the wall because of the capacity of the rubber to absorb the impact energy. Results of this study on single walls are expected to be further extended to global masonry structures, account taken for the influence of actual boundary conditions of the wall.
\end{abstract}




\section{INTRODUCTION}

\subsection{General context}

Unreinforced load-bearing masonry structures are traditionally used for single family houses. Nevertheless, recent improvements of their mechanical properties and better control of their global behaviour have led to the extension of their range of application to multi-storey buildings up to 5-6 levels, which are particularly used for apartments [1]. This kind of buildings generally requires a good acoustic insulation level to fulfil the standards in terms of individual comfort. A convenient and validated solution consists in placing a rubber layer at the bottom and/or top of each wall to prevent acoustic bridges (see Figure 1).

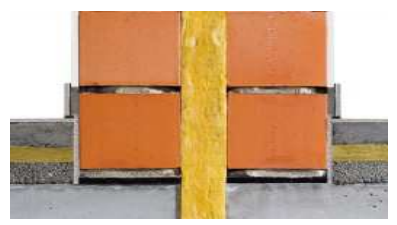

Figure 1 - Acoustic solution (Wienerberger)

The influence of this technical solution on the seismic behaviour of multi-storey unreinforced masonry structures optimized for acoustic performances is however questionable, even in the case of moderate seismic action. Indeed, the rubber layers are likely to modify the stiffness and resistance of the structural elements as well as the boundary conditions of the walls. In this perspective, shaking table tests have been carried out at the Earthquake and Large Structures Laboratory (EQUALS) of the University of Bristol, in the framework of the European project SERIES. The research program aims at a better understanding of the seismic behaviour and of the consequences of the use of rubber layers on the dynamic behaviour of the walls and hence of the global structure. This paper presents a summary of the test specimens, procedures and results, followed by a comparison of the actual experimental behaviour with respect to theoretical rocking models assuming a rigid-body behaviour of the wall. The assumptions on the criterion defining the initiation of the rocking motion and on the restitution coefficient are also discussed.

\subsection{Description of the specimens}
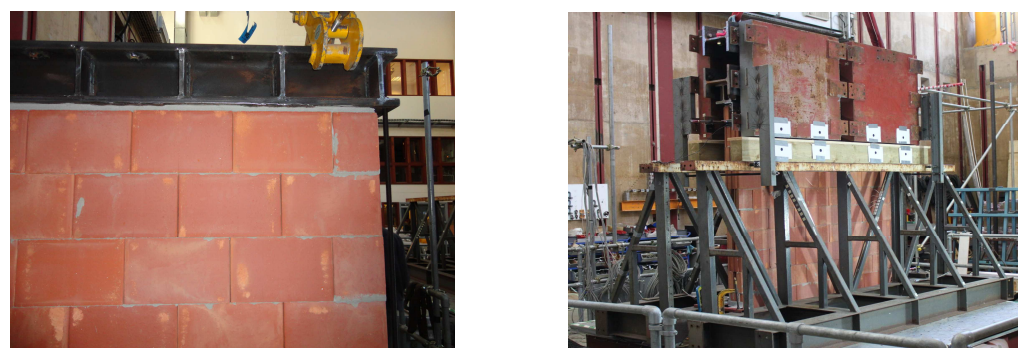

Figure 2 - View of a specimen

Studied specimens are single walls constituted by thin-bed layered clay masonry with empty vertical joints (see Figure 2). Two of them have an aspect ratio close to 1, while it is close to 0.4 for the other two. Exact dimensions of the walls are the following :

- $\quad$ Length $\times$ Height $\times$ Width $=2.1 \mathrm{~m} \times 1.8 \mathrm{~m} \times 0.138 \mathrm{~m}$ (long wall)

- $\quad$ Length $\times$ Height $\times$ Width $=0.72 \mathrm{~m} \times 1.8 \mathrm{~m} \times 0.138 \mathrm{~m}$ (short wall)

The block dimensions are length $x$ height $x$ width $=300.0 \mathrm{~mm} \times 188.0 \mathrm{~mm} \times 138.0 \mathrm{~mm}$ 
Mechanical characteristics of the units and masonry are the following ones:

- $\quad$ Normalised compressive strength of units (EN 772-1 Annex A)

$$
f_{b}=13.0 \mathrm{~N} / \mathrm{mm}^{2}
$$

- $\quad$ Measured characteristic masonry compressive strength (EN 1052-1)

$$
f_{k}=5.6 \mathrm{~N} / \mathrm{mm}^{2}
$$

- $\quad$ Characteristic compressive strength (NBN-EN 1996-1-1)

$$
f_{k}=3.9 \mathrm{~N} / \mathrm{mm}^{2}
$$

A 5-tons mass is placed at the wall top to emulate the structural floor load, with due consideration to the shaking table capacities and to the common range of compression level in masonry structures. The instrumentation layout of the tested specimens and safety arrangements are extensively described in $[2,3]$.

\section{DESCRIPTION AND SUMMARY OF THE TEST RESULTS}

The experimental procedure includes two different types of tests. The first type is performed in order to characterize the specimen dynamic properties (natural frequency, damping) on the base of a "white noise" excitation. The second type consists in seismic test stricto sensu, using an artificially generated seismic input signal consistent with Eurocode 8 spectrum, with an acceleration level increased step-by-step. The PGAs measured during the seismic tests are given in Table 1. Details of the testing procedures and extensive analysis of the results are available in $[2,3]$. The main information and results are summarized in the present paper and the main conclusions are recalled.

\begin{tabular}{ccccc}
\hline No Test & $\begin{array}{c}\text { Long wall } \\
\text { without rubber }\end{array}$ & $\begin{array}{c}\text { Long wall } \\
\text { with rubber }\end{array}$ & $\begin{array}{c}\text { Short wall } \\
\text { without rubber }\end{array}$ & $\begin{array}{c}\text { Short wall } \\
\text { with rubber }\end{array}$ \\
\hline 1 & 0.0393 & 0.0426 & 0.0413 & 0.0417 \\
2 & 0.0777 & 0.0901 & 0.0654 & 0.0604 \\
3 & 0.0777 & 0.0877 & 0.0635 & 0.0607 \\
4 & 0.1583 & 0.1871 & 0.0867 & 0.0803 \\
5 & 0.2387 & 0.2784 & 0.1356 & 0.1235 \\
6 & 0.3230 & 0.3556 & 0.331 & 0.1278 \\
7 & 0.4496 & 0.4567 & 0.1784 & 0.1709 \\
8 & 0.5716 & 0.5692 & 0.1869 & $/$ \\
9 & 0.6878 & 0.6392 & 0.2336 & $/$ \\
\hline
\end{tabular}

Table 1 - Measured PGA [g]

In practice, for each specimen, the testing sequence starts with a "white noise" test. Then, the procedure consists in an alternation of seismic and "white noise" tests in the perspective of studying the effects of the earthquake action on the specimen in terms of degradation of the dynamic properties (natural frequency and damping).

\subsection{White noise test results}

White noise tests are mainly useful to characterize the specimens in terms of natural frequencies, modal shape and damping ratio. The evolutions of the natural frequencies and the damping ratio are represented respectively in Figure 3 and Figure 4. Note that seismic tests have been duplicated at selected acceleration levels to study the effects of repeated earthquakes. 

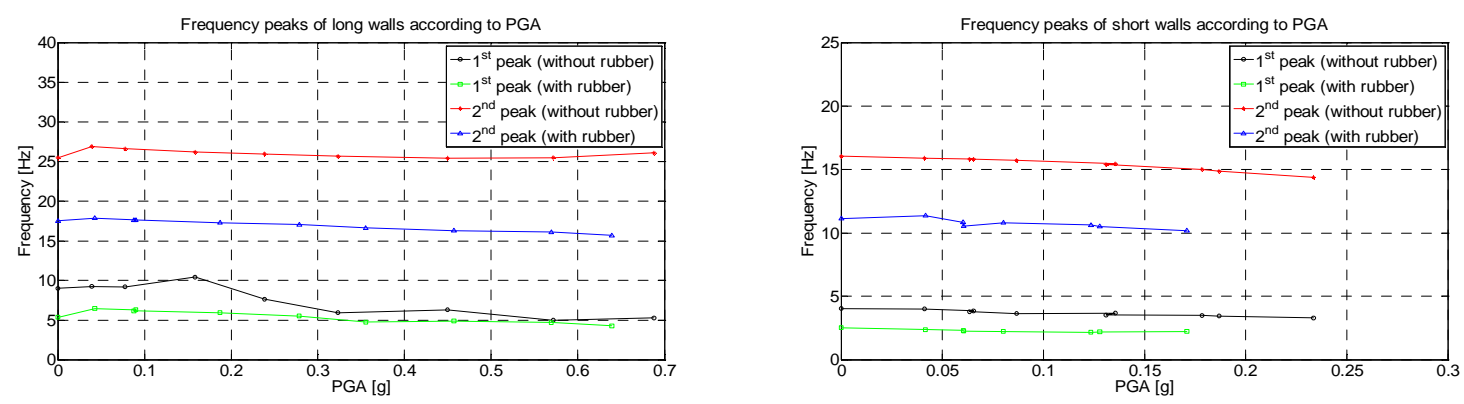

Figure 3 - Natural frequencies

Walls without rubber layers are characterized by higher values in terms of natural frequencies. The difference is about $30 \%$ to $40 \%$ for undamaged situations. When going through the testing sequence, a decrease of the natural frequency is observed for each wall. This observation can be explained by the deterioration of the specimens. Walls without rubber present however a more important frequency drop translating a higher degree of damage for a same ground acceleration level.
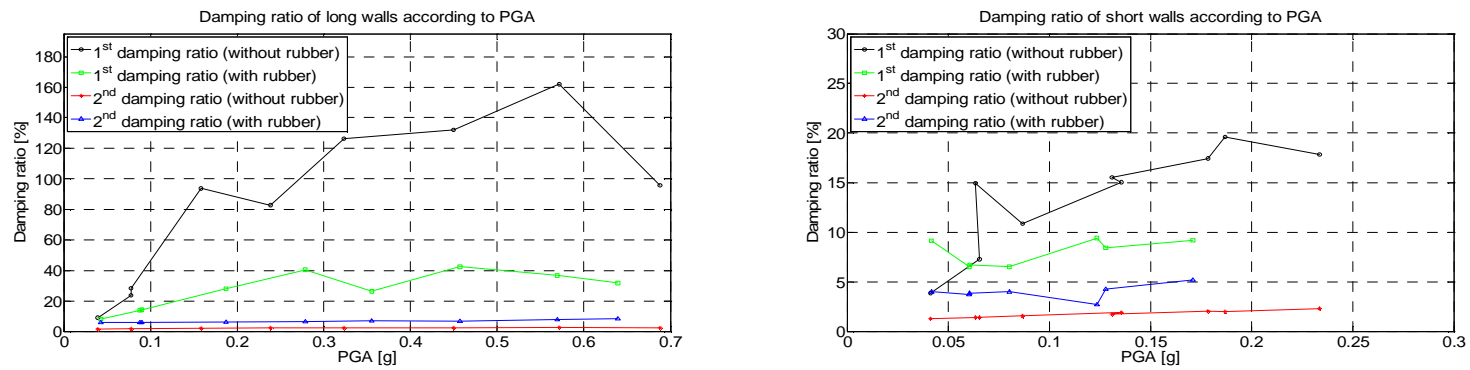

Figure 4 - Damping ratio

An increase of the acceleration level results also in an increase of the damping ratio. Although this increase occurs for each specimen, it is more important for the first mode of walls without rubber devices, especially for the longer one. Some measurements are however questionable since their values are as high as $100 \%$. This could be explained by a poor accuracy of the procedure used to determine the damping ratio.

The influence of rubber layers can also be clearly highlighted. One can firstly think that the presence of acoustic insulation devices is unfavourable for the structure because it results in lower natural frequencies and hence in a higher flexibility and in larger displacements. Nevertheless, it appears that these devices have positive effects. Indeed, even if the seismic shakes affect the wall properties, rubber devices mitigate the frequency drop and limit the damping increase. As proposed in [3], a possible and convenient explanation is due to an essentially different behaviour. With the rubber layers, the assumption of rigid support is no more valid and the wall has to be considered as resting on an elastic foundation. Therefore, the instantaneous energy dissipation occurring when the wall passes through the vertical position is lower and the damages due to a less strong impact are thus less important.

\subsection{Seismic test results}

A major output of the seismic tests is the possible measurement of the compressive length, this so-called compressive length being actually the contact length at the interface between the wall and its foundation. This parameter is indeed the main one used in the design of walls and the assessment of their overturning resistance according to classical static equivalent design methodologies such as suggested by the Eurocode 6 [4]. 


\subsubsection{Design method of Eurocode 6}

The verification methodology proposed by the Eurocode 6 is a static equivalent one based on the static equilibrium of an element submitted to a combination of normal force and bending moment due to a horizontal shear. For a same loading scheme, the value of the compressive length can however be different, depending on the assumption of the shape of the normal stress distribution at the wall-foundation interface. It is indeed possible to consider for instance a constant $(2, a)$ or a linear $(2, b)$ stress distribution. These two assumptions respectively lead to the following formulas to estimate the compressive length $L_{c}$ :

$$
\begin{gathered}
M=V . H \\
L_{c}=\left\{\begin{array}{cc}
0 & \text { if } e \geq L / 2 \\
2 .\left(\frac{L}{2}-e\right) & \text { if } e \leq L / 2 \wedge e \geq 0 \\
L & \text { if } e=0
\end{array}\right. \\
L_{c}=\left\{\begin{array}{cc}
0 & \text { if } e \geq L / 2 \\
3 .\left(\frac{L}{2}-e\right) & \text { if } e \leq L / 2 \wedge e \geq L / 6 \\
L & \text { if } e \leq L / 6
\end{array}\right.
\end{gathered}
$$

\subsubsection{Experimental results and comparisons}

The methodology used to derive the compressive length from the direct test measurements is developed in [2] and is based on the assumption that the base section of the wall remains plane. Illustrative values are given in Table 2 and compared with values calculated according to the Eurocode procedure for selected acceleration levels. Figure 5 shows the complete evolution of the experimental compressive length according to the acceleration level.

\begin{tabular}{cccccccc}
\hline Specimen & $\begin{array}{c}\text { Acceleration } \\
\text { level }[\mathrm{g}]\end{array}$ & \multicolumn{2}{c}{ Compressive length } & \multicolumn{2}{c}{ Assessment (linear) } & \multicolumn{2}{c}{ Assessment (constant) } \\
& {$[\%]$} & {$[\mathrm{mm}]$} & {$[\%]$} & {$[\mathrm{mm}]$} & {$[\%]$} & {$[\mathrm{mm}]$} \\
\hline Long wall & 0.04 & 83.20 & 1747.2 & 100.00 & 2100 & 82.86 & 1740 \\
without & 0.15 & 47.15 & 990.15 & 57.23 & 1201.9 & 35.71 & 750 \\
$\quad$ rubber & 0.66 & 0.03 & 0.5 & 0 & 0 & 0 & 0 \\
\hdashline Long wall & 0.04 & 100.00 & 2100.0 & $/$ & $/$ & $/$ & $/$ \\
$\quad$ with & 0.19 & 67.20 & 1411.2 & $/$ & $/$ & $/$ & $/$ \\
rubber & 0.64 & 0.05 & 1.1 & $/$ & $/$ & $/$ & 1 \\
\hline Short wall & 0.01 & 77.01 & 554.5 & 100.00 & 720 & 87.50 & 630 \\
without & 0.02 & 58.70 & 422.6 & 100.00 & 720 & 75.00 & 540 \\
$\quad$ rubber & 0.15 & 14.37 & 103.5 & 0 & 0 & 0 & 0 \\
\hline Short wall & 0.04 & 100.00 & 720.0 & $/$ & $/$ & $/$ & $/$ \\
$\quad$ with & 0.06 & 100.00 & 720.0 & $/$ & $/$ & $/$ & $/$ \\
rubber & 0.17 & 32.13 & 231.3 & $/$ & $/$ & $/$ & $/$ \\
\hline
\end{tabular}

Table 2 - Illustrative values of compressive length 


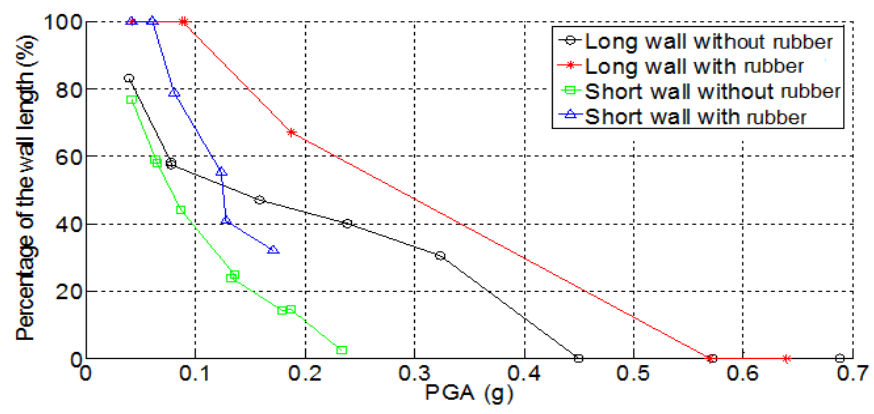

Figure 5 - Compression length

Comparison between measurements and theoretical predictions yields the following comments. For low acceleration level, the measured values are intermediate between the theoretical values obtained assuming the two different shapes of stress distribution. For higher acceleration level, the design method underestimates the compressive length. Note that, contrary to what is assumed in an equivalent static procedure, a zero value for the compressive length doesn't necessary mean a collapse of the wall, but can be associated to a rocking motion. The influence of rubber devices on the compressive length is favourable. This length is indeed larger for a same acceleration level in presence of acoustic insulation devices.

\subsection{Classification of the test results}

A sorting of the tests is proposed based on a comparison of the rotations measured at the bottom and top of the wall. Three different situations are identified.

The first situation appears for the seismic tests with a low level of acceleration, where a significant difference between rotations at the wall bottom and top is observed, as illustrated in Figure 6 for the first seismic test on the short wall without rubber devices. In this case, it is shown in [5] that the specimens can accurately be modelled as a cantilever beam (provided shear deformability is correctly accounted for). Among the present set of experimental results, this assumption is shown to be valid for the first three seismic tests. Comparisons between measurements and modelling results are carried out in [5]. This range and the associated modelling assumption are mainly useful for assessing damage limit states at low acceleration level.

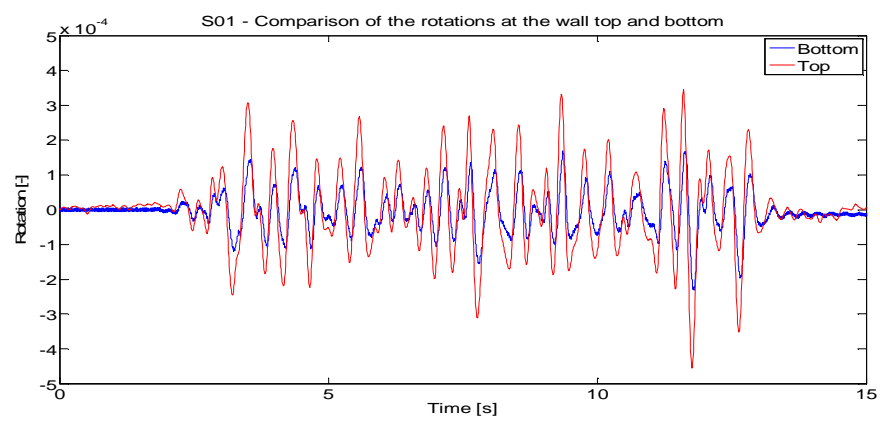

Figure 6 - Bottom and top rotations for a low level of acceleration

The second case corresponds to situations where quasi-equal rotations are measured at the top and at the bottom. This is observed when the acceleration level is rather high (tests S07, S08, S09). Figure 7 illustrates the situation for the seismic test S08 on the short wall without rubber devices. In this case, the specimen can be considered as a rigid body rocking on its support. Section 3 of this paper will focus specifically on the modelling of this situation. This range is of prime interest for the evaluation of the ultimate limit state. 


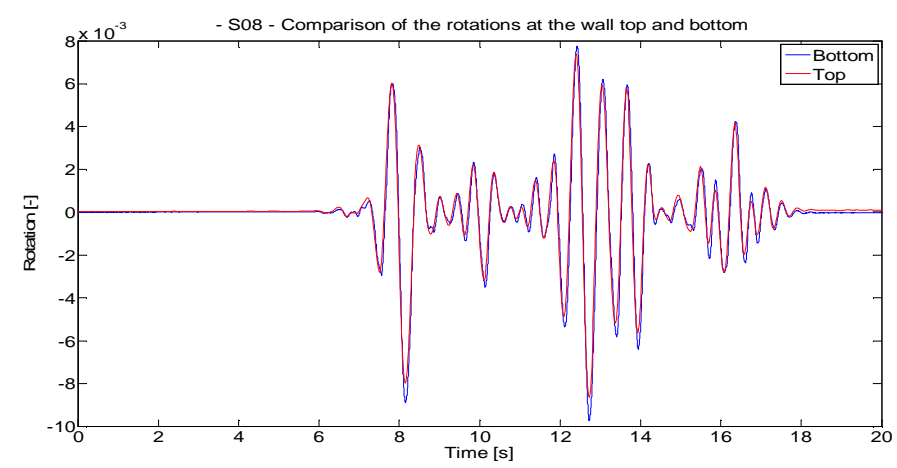

Figure 7 - Bottom and top rotations for a high level of acceleration

The third situation is a hybrid one and corresponds to tests with an intermediate acceleration level (S04, S05 and S06). A proper modelling would thus require combining a simple cantilever with a rocking model. It is felt however of a more limited practical interest.

\section{DEVELOPMENT OF A THEORETICAL ROCKING MODEL AND COMPARISONS WITH TESTS RESULTS}

\subsection{Description of the reference theoretical model}

\subsubsection{Basic equations}

The theoretical model used to carry out the comparisons with the experimental results is derived from the historical reference model developed by Housner [6]. This model has been initially developed to study the oscillations of a rigid body standing on a rigid support and is based on the resolution of the equation of motion deduced from the theorem of angular momentum :

$$
\dot{H}_{O}=M_{O}
$$

where $H_{O}$ is the angular momentum relative to $\mathrm{O}$

$M_{O}$ is the resultant of moments of external forces relative to $\mathrm{O}$.

In the reference version of the model, the gravity load is the specific weight of the body and is applied at its centre of gravity, as well as the inertial forces. The actual configuration of the tested specimens requires adjustments of this reference model in order to take into account the fact that :

- The specific weight of the wall can be reasonably neglected since it is less than $10 \%$ of the additional mass lying on the top of the wall;

- The main gravity load and the inertial forces are consequently acting at the top of wall.

The model considers thus a rigid body on a rigid support, with $\mathrm{H}$ and $\mathrm{B}$ being respectively the height and the length of the body. The rigid body is characterized by the angle $\alpha=\tan (B / 2 H)$. This angle characterizes the maximum rotation of the block beyond which the body turns over in static conditions under the effect of the gravity only. The two lower corners of the body are denoted $\mathrm{O}$ and O'. As it is assumed that the block and the support are both rigid, the oscillations are alternatively around $\mathrm{O}$ and $\mathrm{O}$ '. The angle between the body and the vertical is called $\theta$ and is the main kinematic unknown of the problem. $\theta$ is positive in clockwise rotation. A last assumption is to consider the coefficient of friction large enough for the risk of sliding to be neglected. 


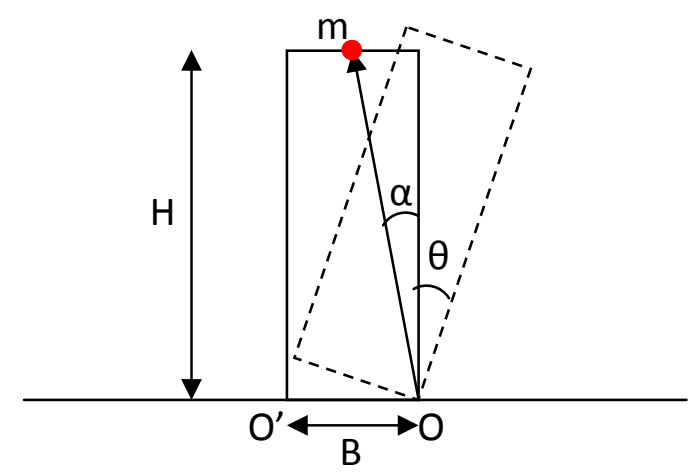

Figure 8 - Sketch of the model configuration

In Figure 8, the distance between a corner and the application point of the gravity load is denoted $R(2)$.

$$
R=\sqrt{H^{2}+(B / 2)^{2}}
$$

In Equation (1), the angular momentum relative to $\mathrm{O}$ is the result of the multiplication of the moment of inertia $I_{O}$ calculated in $\mathrm{O}$ by the time-derivative $\dot{\theta}$. As the contribution of the specific weight of the wall is neglected, it follows :

$$
\begin{gathered}
\dot{H}_{O}=I_{O} . \ddot{\theta} \\
\text { with } \quad I_{O}=\rho \int_{A}\left(x^{2}+y^{2}\right) d A+m R^{2}=m R^{2}
\end{gathered}
$$

In presence of a seismic action, the resultant of moments of external forces relative to $\mathrm{O}$, i.e. $M_{O}$, is due to the gravity load and to the inertial force, yielding :

$$
M_{O}=-m g R \sin (\alpha \cdot \operatorname{sign} \theta-\theta)+m \ddot{u}_{g} R \cos (\alpha \cdot \operatorname{sign} \theta-\theta)
$$

where $m[\mathrm{~kg}]$ is the dead load

$g\left[\mathrm{~m} / \mathrm{s}^{2}\right]$ is the gravity

$\ddot{u}_{g}\left[\mathrm{~m} / \mathrm{s}^{2}\right]$ is the seismic acceleration

\subsubsection{Numerical resolution}

The resolution of the equation of motion is carried out using a standard Newmark integration scheme, under the assumption of constant acceleration $(\beta=1 / 4, \delta=1 / 2)$. The following equations (6), (7) and (8) are thus implemented to determine the time evolution of the angle $\theta$.

$$
\begin{gathered}
\ddot{\theta}_{n+1}=-\frac{m g R}{I_{O}}\left[\sin \left(\alpha \cdot \operatorname{sign} \theta_{n}-\theta_{n}\right)+\frac{\ddot{u}_{g, n+1}}{g} R \cos \left(\alpha \cdot \operatorname{sign} \theta_{n}-\theta_{n}\right)\right] \\
\dot{\theta}_{n+1}=\dot{\theta}_{n}+\left[(1-\delta) \cdot \ddot{\theta}_{n}+\delta \cdot \ddot{\theta}_{n+1}\right] \cdot \Delta t=\dot{\theta}_{n}+0 \cdot 5\left[\ddot{\theta}_{n}+\ddot{\theta}_{n+1}\right] \cdot \Delta t \\
\theta_{n+1}=\theta_{n}+\dot{\theta}_{n} \cdot \Delta t+\left[\left(\frac{1}{2}-\beta\right) \cdot \ddot{\theta}_{n}+\beta \cdot \ddot{\theta}_{n+1, l i n}\right] \cdot \Delta t^{2} \\
=\frac{\theta_{n}+\dot{\theta}_{n} \cdot \Delta t+\frac{\Delta t^{2}}{4}\left[\ddot{\theta}_{n}-\frac{m g R}{I_{0}}\left(\alpha \operatorname{sign} \theta_{n+1}+\frac{\ddot{u}_{g, n+1}}{g}\right)\right]}{1-\frac{m g R}{I_{0}} \frac{\Delta t^{2}}{4}}
\end{gathered}
$$




\subsubsection{Initiation of the rocking motion}

The rocking motion of the wall is initiated as soon as the moment due to the inertial force is higher than the restoring force moment of the gravity load. The equilibrium is expressed with respect to the corner $\mathrm{O}$ or $\mathrm{O}^{\prime}$ according to the assumption of a rigid body placed on a rigid support. Therefore, a rocking criterion $R C$ can be written as follows :

$$
R C=\frac{m \ddot{u}_{g} H}{m g \frac{B}{2}}>1
$$

As long as the condition (9) is not verified, no rocking motion is initiated and the angle $\theta$ remains equal to zero.

\subsubsection{Coefficient of restitution}

When the body oscillation switches from a rotation around one of its corners to a rotation around the other one, it has to pass through its original resting vertical position. This transition is actually associated with an impact since the body hits the support. This impact obviously dissipates energy. The approach proposed by Housner to account for the dissipation is to consider the conservation of angular momentum with various assumptions (see [7]). According to this, a reduction factor $e$ is defined and has to be applied to the velocity of the wall before the impact to obtain the velocity right after this impact. The reduction factor is given by :

$$
e=1-2 \frac{m R^{2}}{I_{O}} \sin ^{2} \alpha
$$

where $\alpha$ is the angle drawn in Figure 8. In this way, the incoming velocity is reduced each time the angle $\theta$ changes its sign.

\subsection{Predictions with the reference theoretical model}

The theoretical model described above is first crudely applied to perform a direct simulation of the seismic tests S07, S08 and S09 for the walls without rubber layers. The input data for the theoretical prediction is the shaking table acceleration signal as measured during the test.

Figures 9, 10 and 11 shows the evolution of the Rocking Initiation Criterion calculated according to Equation (9) with the theoretical predictive model. Left-hand side and right-hand side graphs correspond respectively to the short and long walls. It is observed in Figure 9 and Figure 10 that the Rocking Criterion is never overcoming a unit value for tests S07 and S08, translating the fact that the theoretical model is predicting a non-occurrence of rocking motion. On the other hand, Figure 11 shows a criterion exceeding the unit, and thus predicting the initiation of a rocking motion, at about 4 seconds for the short wall (left) and at about 10 seconds for the long wall (right).

Together with the time-evolution of the rocking criterion, all three figures also present the time-evolution of the measured rotation of the wall illustrating the actual rocking behaviour observed during the tests, with a significant motion occurring for tests S07 and S08 although not predicted. It can thus already be concluded that a further modification of the rocking criterion is required. The impossibility of the theoretical model to predict the initiation of motion is at this stage identified as a consequence of the strong assumption of a perfectly rigid body lying on a perfectly rigid foundation and will be discussed in section 3.3.1. 

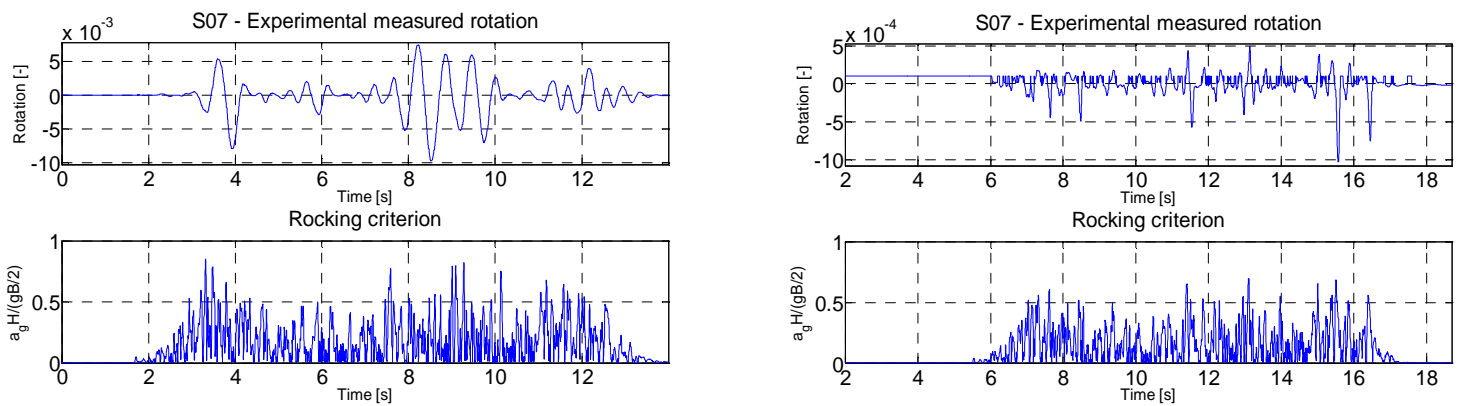

Figure 9 - Rocking criterion for short (left) and long (right) walls without rubber devices during seismic test $\mathrm{S07}$
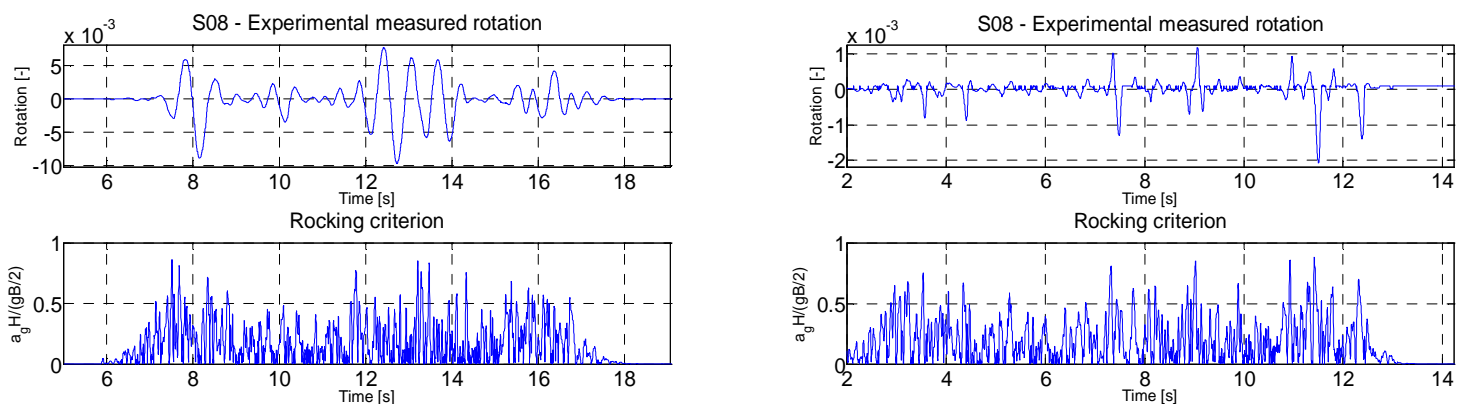

Figure 10 - Rocking criterion for short (left) and long (right) walls without rubber devices during seismic test S08
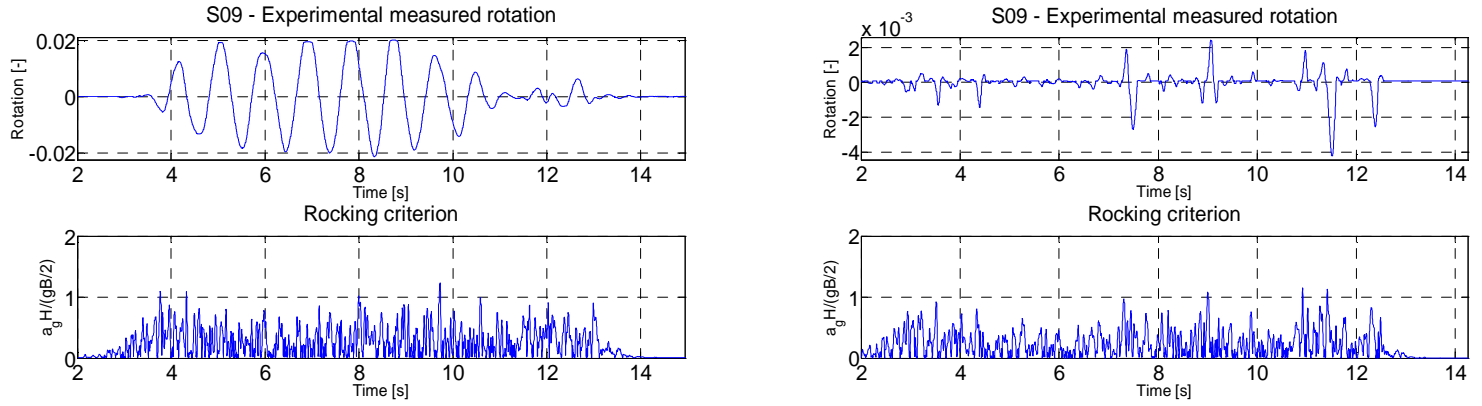

Figure 11 - Rocking criterion for short (left) and long (right) walls without rubber devices during seismic test $\mathrm{S} 09$

As a further comparison, Figure 12 compares the rotation calculated by the theoretical model with those observed from tests S09, for which the rocking criterion is predicted a motion. This figure evidences the two main issues faced by the theoretical model.

The first one is related with the definition of the rocking criterion. Indeed, the initiation of the motion in Figure 12 (right) occurs at about 10 seconds, whereas the measurements are catching a rotation of the wall from 2 seconds. This observation is in line with the conclusions of tests S07 and S08 about a too conservative rocking criterion.

The second issue deals with the coefficient of restitution. In Figure 12 (left), it is obvious that the model is not able to reach the level of rotation measured during the test $(t \approx 5 \mathrm{~s})$, while it predicts a non-observed amplification of the oscillations at the end of the signal $(t>10 \mathrm{~s})$. These observations can be felt as due to a respectively too low or too high estimate of the coefficient of restitution. 

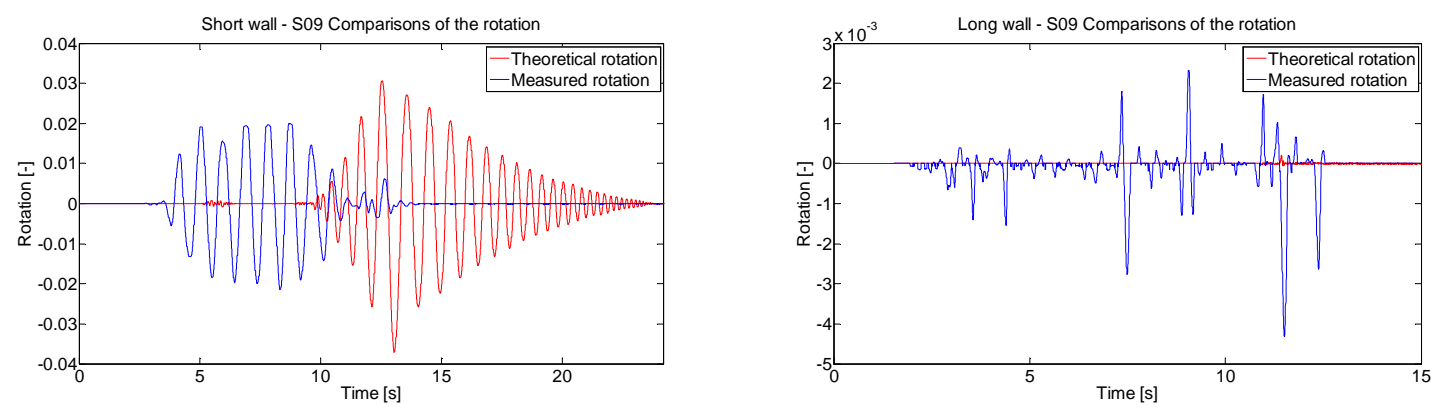

Figure 12 - Comparison between model and experimental results (S09)

\subsection{Improvements of the reference model}

In conclusion of the previous section, it comes out that the crude use of a basic rocking model is not suitable for walls. The two main parameters that could be adjusted to tune the model for the specific situation considered in the present study are respectively the condition initializing the rocking motion and the estimate of the restitution coefficient. Possible adjustments are proposed in the following sections.

\subsubsection{Initiation of the rocking motion}

As the contact between the masonry wall and its foundation cannot be considered as perfectly rigid, the assumption of a rigid body rotation around its edge considered as a single dimensionless point is certainly not valid.

It is generally assumed that masonry structural elements have no tensile strength. Thus, the equilibrium is performed according to a static equivalent method such as for instance the approach previously described in 2.2.1. This equilibrium implicitly considers that the base section of the wall remains plane, from which a strain then a stress distribution can be derived. The proposed modification of the rocking initiation criterion consists in considering that, when the motion starts, the rotation doesn't occur around the edge of the wall but around the point of zero stress, and thus zero strain, calculated according to $\S 2.2 .1$. It yields:

$$
R C=\frac{m \ddot{u}_{g} H}{m g\left|\frac{B}{2}-L_{C}\right|}>1
$$

where $L_{c}$ is the compressive length calculated with the assumption of a linear distribution of stresses.

With this updated definition of the rocking criterion, the model is predicting the initiation of a rocking behaviour for all tests considered in the study (i.e. S07 to S09 for both aspect ratios of the walls). Figure 13 illustrates this for test S07 of the short wall. In this figure, the criterion calculated by Equation (9) remains below the motion initiation threshold, whereas the modified one calculated by Equation (11) is now sufficient to initiate the motion. 


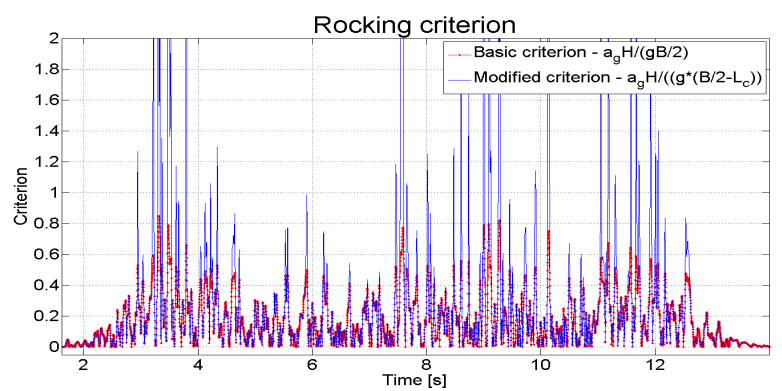

Figure 13 - Rocking criterion for short wall during seismic test S07

In order to keep a fully consistent formulation, the modification of the rocking criterion in terms of position of reference fix point also influences the constitutive equations of the model. Indeed, once the motion starts, the rotation point should now be assumed to be calculated in the same way as when estimating the rocking initiation criterion. This is also consistent with the test measurements summarized in Table 2 and showing that the compressive length generally does not reduce to a single point, even in the case of a slender wall under large acceleration level.

In practice, it implies that the rotation point is likely to vary at each time step (see Figure 14). As a consequence, the angle $\alpha$ is no more a constant, as well as the distance $R$ or the coefficient of restitution $e$, these last two parameters being indeed function of the angle $\alpha$. The values at time-step $n$ are then :

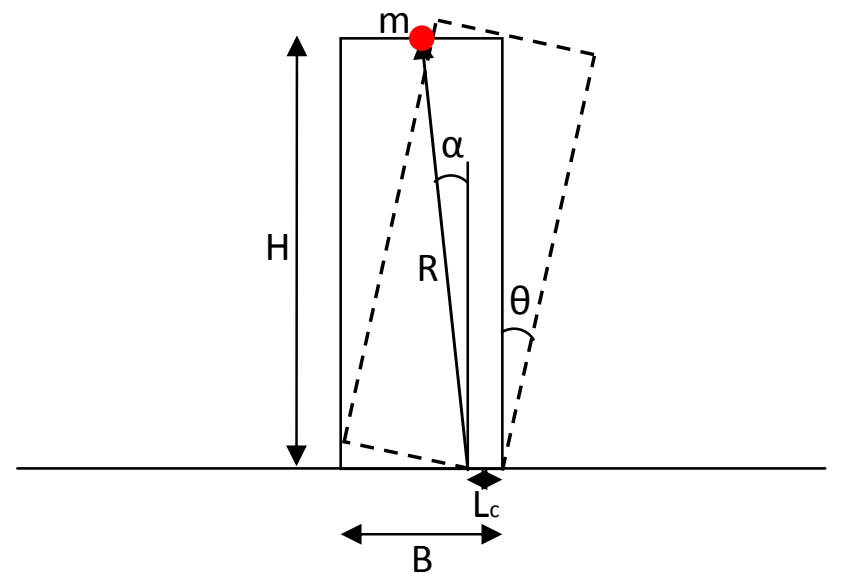

Figure 14 - Updated sketch of the model configuration

$$
\begin{gathered}
\tan \alpha_{n}=\frac{\left|\frac{B}{2}-L_{C, n}\right|}{H} \\
R_{n}=\sqrt{H^{2}+\left|\frac{B}{2}-L_{C, n}\right|}=\frac{H}{\cos \alpha_{n}} \\
e=1-2 \frac{m R_{n}^{2}}{I_{O, n}} \sin ^{2} \alpha_{n}
\end{gathered}
$$

This only modification is however not sufficient alone to get accurate results, although the general trend of the calculated motion is already better in the initial stage of the simulation when compared to experimental results. This is illustrated in Figure 15, where the model with modified reference point is run for the S09 short wall. 


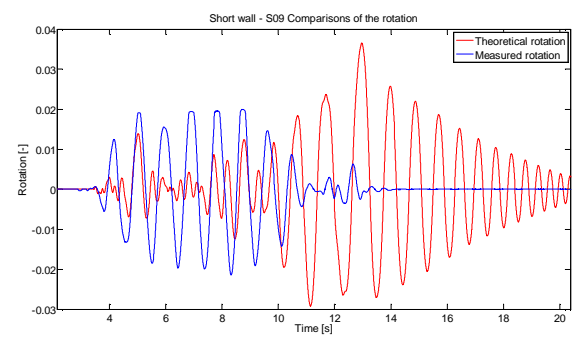

Figure 15 - Comparison between model and experimental results

\subsubsection{Restitution coefficient}

The formulation of the restitution coefficient used in the basic reference model is the one proposed by [7] deduced from the theory developed by Housner. An alternative formulation is however proposed by [8], also on the base of the Housner theory, that can in the present case be further simplified according to the specific expression of $I_{O}$ :

$$
e_{n}=1-\frac{m R_{n}^{2}}{I_{O, n}} \sin ^{2} \alpha_{n}=1-\sin ^{2} \alpha_{n}
$$

This shows that different options are actually possible regarding the coefficient of $\sin ^{2} \alpha$ in the expression of $e_{n}$. In the case of a real masonry wall, the restitution coefficient is very likely to be influenced by the level of degradation of the material, while this level of degradation at a given impact is also likely to depend on the amplitude of the motion during the previous oscillation. The largest is the amplitude, the stronger is the impact, the higher is the dissipation and hence the lower is the restitution coefficient. This dependency is assumed to be represented by the ratio of the maximum rotation calculated during the oscillation preceding the considered impact $\theta_{\text {max }, n}$ normalized by the very maximum rotation calculated since the beginning of the motion $\theta_{\text {max }, a b s}$, yielding:

$$
e_{n}=1-\frac{\theta_{\text {max }, n}}{\theta_{\text {max }, a b s}} \sin ^{2} \alpha_{n}
$$

A second modification is introduced to take into account a lower level of dissipation observed as soon as the seismic input is stopped. Indeed, in case the same coefficient is used during the whole simulation, an unexpected amplification of the response is predicted right after the occurrence of the earthquake (see Figure 15). Therefore it is suggested to use two different expressions of the restitution coefficient for the forced vibration and for the free vibration motions. Eq. (16) is considered for the forced vibrations, while an empirically modified value is used for the free vibration, as suggested in Eqs. (17).

$$
e_{n}= \begin{cases}1-\frac{\theta_{\max , n}}{\theta_{\max , a b s}} \sin ^{2} \alpha_{n} & \text { if } \ddot{u}_{g} \neq 0 \\ 1-\frac{1}{2} \frac{\theta_{\max , n}}{\theta_{\max , a b s}} \sin ^{2} \alpha_{n} & \text { if } \ddot{u}_{g}=0\end{cases}
$$

A final dependency on the wall aspect ratio is introduced on the base of the test observations in order to improve the fitting of the results for the long wall. Indeed, in this latter case, the rocking is clearly not the one of a pure rigid body, as evidenced by the higher values of the compressive length given in Table 2. It is thus assumed that, all other conditions being 
identical, i.e. for similar values of the parameters in Eq. (17), the energy dissipated by the long wall should be lower than by the shorter one. A reduction factor is then introduced to account for this effect. An empirical approach by progressive fitting of the numerical results with respect to the experimental data leads to a dependency proportional to the square of the proportion of the aspect ratios, yielding Eq. (18), where $A_{0}$ is a constant to be calibrated:

$$
e_{n}=\left(\frac{A_{0}}{\text { Aspect_Ratio }}\right)^{2}\left(1-\frac{\theta_{\max , n}}{\theta_{\max , a b s}} \sin ^{2} \alpha_{n}\right)
$$

\subsection{Predictions of the modified theoretical model}

Theoretical predictions are first compared with experimental results for the shorter wall in Figure 16, Figure 17 and Figure 18 (left). For this specimen, the model predictions and test measurements match reasonably well, in particular in terms of frequency content and prediction of the strong motions. The time-evolution is however slightly different with a relative difference of the maximum rotation equal to about $30 \%$ in the worst case. Another discordance appears at the end of the test, once the acceleration signal comes down to zero (for $\mathrm{t}>11 \mathrm{~s}$ for S07 and S09, t > 14s for S08). This could be explained by the influence of the shaking table itself which modifies artificially the damping through its breaking system. Indeed, the measurements show a renewed increase of the rotation, while the theoretical results remain close to zero. Except for these observations, the most important phase of strong motion appears to be well approached by the model. This phase corresponds to the motion between the fourth and the tenth seconds for S07 and S09 tests and between the eighth and fourteenth for S08 test. In this latter, the correspondence is less good, but the measurements are actually disturbed by the specimen hitting the safety arrangements. Figure 17 (left) shows a asymmetric rocking behaviour with higher values of positive rotations, which is in accordance with the model predictions.

Comparisons for the longer wall are plotted in Figure 16, Figure 17 and Figure 18 (right). for seismic test S07, S08 and S09 respectively. The fitting of the theoretical curve with the experimental one is pretty accurate in terms of predicting the occurrences of peak rotations, although the orders of magnitude of these peaks are generally overestimated by the theoretical model.
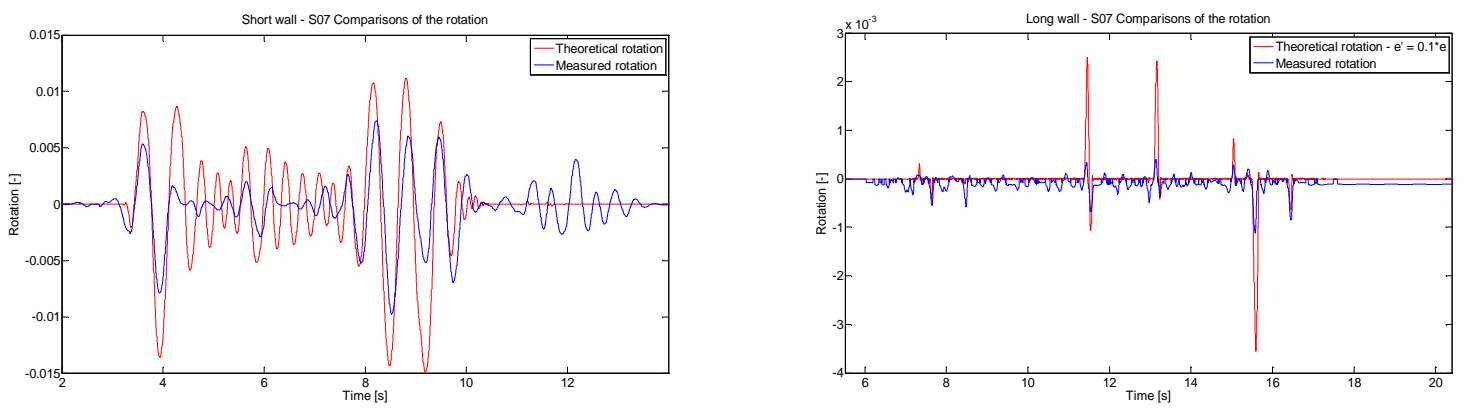

Figure 16 - Results for S07 (short wall : left - long wall right) 

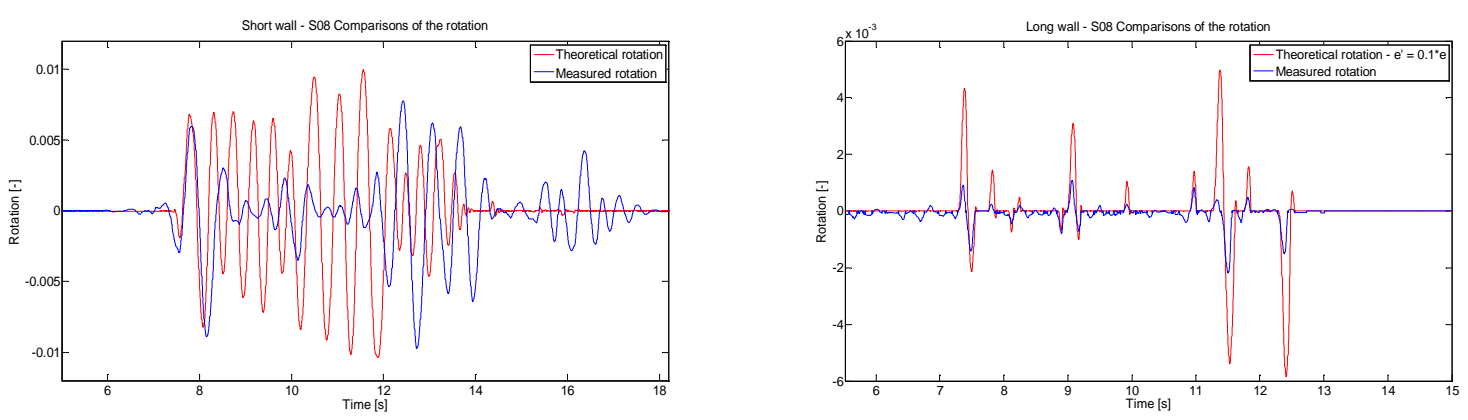

Figure 17 - Results for S08 (short wall : left - long wall right)
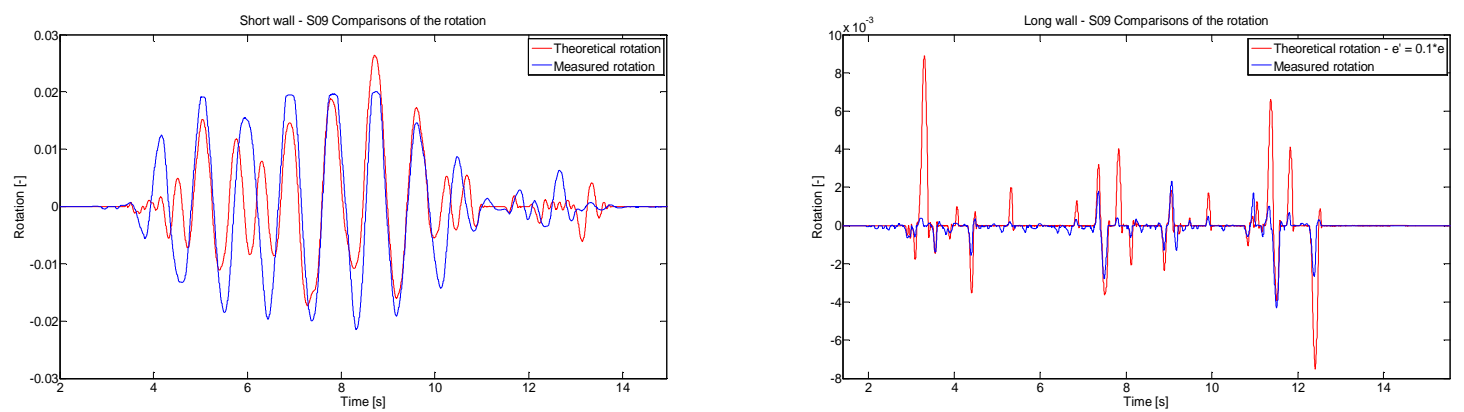

Figure 18 - Results for S09 (short wall : left - long wall right)

Results of the experimental tests and predictions of the model can also be compared in terms of the uplifting of the wall. Let's denote by "lifted length", the part of the wall which is no longer in contact with the support. Its time-evolution is drawn in Figure 19 and Figure 20 for the tests S07 and S09 respectively on short wall (left) and long wall (right). The results provided by the theoretical model assume that the wall is either straight in vertical position (lifted length equal to zero) or completely uplifted. Test and predictive values are in good agreement in the case of the short wall, especially for the seismic test S09. This latter observation was expected since the test conditions were the closest to the model assumptions in terms of measured contact length. In the case of the long wall, the correspondence of the results is not so clear and several differences are observed. Mainly, the model is not able to simulate every single uplifting of the wall. The reason may be the choice of the point of rotation, although further investigation is clearly required in this perspective.
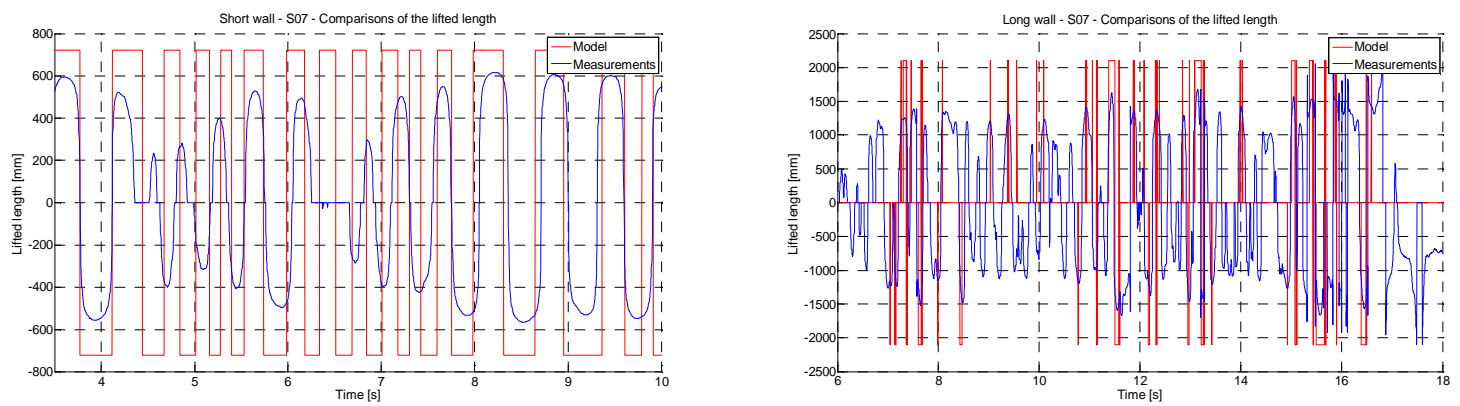

Figure 19 - Comparison of the "lifted length" for the short wall (left) and long wall (right) during seismic tests S07 

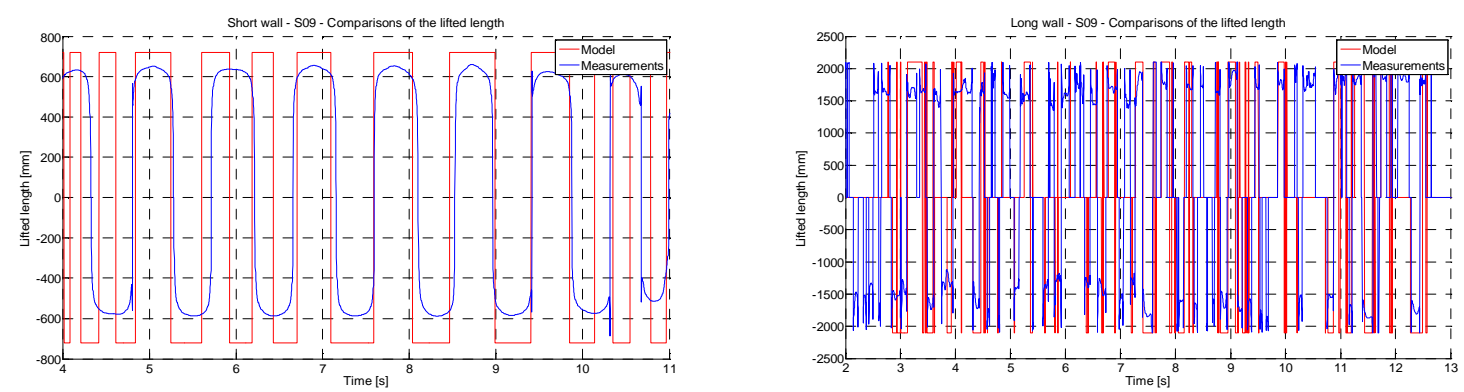

Figure 20 - Comparison of the "lifted length" for the short wall (left) and long wall (right) during seismic tests S09

\section{CONCLUSIONS}

The present paper describes the results of experimental tests on simple unreinforced masonry walls, with glued horizontal joints and empty vertical joints, stressed by a seismic action. In the first part, the paper presents the general test observations, including the consequences of the use of rubber elements on the global behaviour. The following observations are made:

- The natural frequencies of the element are decreased by about $30 \%$ to $40 \%$ in presence of rubber layers.

- A frequency drop and an increase of the damping ratio are observed as the acceleration level goes up. The presence of rubber reduces the drop and the increase of the damping ratio.

- The motion of the specimen can be easily characterized by its compressive length. This latter can be assessed thanks to static equivalent methods when the acceleration level remains low, but is underestimated for higher acceleration levels.

- The use of rubber layers leads to higher compressive length for a same acceleration level compared to similar walls without rubber but results in higher horizontal displacements.

In the second part of the paper, the different test results are classified in three categories. A first group is gathering the tests at a low acceleration level for which the specimen can be modelled as a cantilever beam. A second one corresponds to tests submitted to high acceleration, characterized by a significant rocking behaviour. The last group is a hybrid one, where the specimen behaviour is a mix of a cantilever-like and of a rocking behaviour.

The paper focuses then on the high acceleration levels and presents a modelling of the specimens without rubber layers with the objective of predicting their rocking behaviour. The following conclusions are made:

- Two main parameters influence the general behaviour, namely the rocking initiation criterion and the restitution coefficient.

- The rocking criterion defines the minimum acceleration required to initiate the rocking behaviour, depending on the geometry of the specimen. As the specimen cannot be considered as perfect rigid body, the criterion is a function of the actual compressive length assessed with the assumption of a linear distribution of stresses along the compressive length.

- Modifications of the restitution coefficient have been empirically derived depending on several factors, such as the number of impacts, the amplitude of the rocking motion, the geometry of the specimen and excitation.

Further perspectives cover the investigation of the modelling and behaviour of walls with rubber and the globalization of the theoretical model to study entire buildings composed by walls likely to exhibit a rocking behaviour. 


\section{ACKNOWLEDGEMENTS}

The research leading to these results has received funding from the European Union Seventh Framework Programme (FP7/2007-2013) under grant agreement $\mathrm{n}^{\circ}$ 227887, SERIES. H. Degée also acknowledges the direct support received from F.R.S.-FNRS (Belgian Fund for Research).

\section{REFERENCES}

[1] Stuerz, ESECMaSE - Enhanced Safety and Efficient Construction of Masonry Structures in Europe. Retrieved May 2012, from ESECMaSE: http://www.esecmase.org

[2] C. MORDANT, Contribution to experimental tests on the seismic behaviour of masonry structural elements. Master dissertation, University of Liège, 2012.

[3] C. MORDANT, M. DIETZ, C. TAYLOR, A. PlUMIER, H. DEGEE, Seismic behaviour of thin-bed layered unreinforced clay masonry shear walls including soundproofing elements, Seismic Evaluation and Rehabilitation of Structures: SERIES Workshop. Geotechnical, Geological and Earthquake Engineering series (chapter 6). Alper Ilki and Michael N. Fardis (eds), Springer (in press), 2013

[4] Eurocode 6 : Design of masonry structures - Part 1.1 : Common rules for reinforced and unreinforced masonry structures, 2004

[5] C. MORDANT, M. DIETZ, H. DEGEE, Shaking table tests on unreinforced loadbearing masonry walls - comparison with simple cantilever model, University of Liege internal report, 2013

[6] G. W. HOUSNER, "The behavior of inverted pendulum structures during earthquakes », Bulletin of the Seismological Society of America, Vol. 53, No. 2, February 1963, p.403-417.

[7] L. SORRENTINO, O. ALSHAWA, L.D. DECANINI, The relevance of energy damping in unreinforced masonry rocking mechanisms. Experimental and analytic investigations, Bull Earthquake Eng 9:1617-164, 2011

[8] A. PALMERI, N. MAKRIS, Response analysis of rigid structures rocking on viscoelastic foundation, Earthquake Engng and Struct. Dyn. 37:1039-1063, 2008. 